\title{
Theoretical Investigation on Biological Activity of Imidazole Derivatives [Carbenzim, Mebendazole] by using (DFT) and (PM3) Methods
}

\author{
Amar Tuma Musa ${ }^{1,2, *}$ and Khalida Abaid ${ }^{2}$ \\ ${ }_{1,2}$ Industrial Research and Development Authority, Ministry of Industry and Minerals, Baghdad, Iraq \\ ${ }^{2}$ Department of Chemistry, College of Sciences, Al-Nahrain University, Baghdad, Iraq
}

\begin{tabular}{ll}
\hline \multicolumn{1}{c}{ Article's Information } & \multicolumn{1}{c}{ Abstract } \\
\hline Received: & The theoretical study represents an essential preliminary stage for the start of \\
15.03 .2021 & any industry, as it gives a theoretical description of the properties of compounds \\
Accepted: & (chemical, physical and biological properties) without conducting research to \\
$27-04-2021$ & find out about this and the least cost. Through the theoretical study, we extract \\
Published: & a clear picture of the chemical compounds before starting to manufacture them \\
$27-06-2021$ & to know the extent of their impact on human health and their chemical and \\
Keywords: & biological effectiveness. Using the Density Functional Theory (DFT/B3LYP) with \\
Carbenzim & base 6-311G, through Gaussian 09 program, the optimize geometry, (bond \\
Mebendazole & lengths, angles bond) and vibrational spectra was calculated of the \\
DFT & benzimidazole derivatives [Carbenzim (CZM), Mebendazole (MBZ)]. \\
PM3 & Through orbital charts of HOMO and LUMO to study electronic properties. \\
Log P & The HOMO-LUMO gap was also evaluated for chemical reactivity and \\
QSAR & determination of global reactivity descriptors (Hardness ( $\eta$, Softness (S), \\
HOMO & Electrophilicity ( $\omega$ ), Chemicalpotential ( $\mu$ ), Electronegativity $(x)$ ) that defines \\
LUMO & compunds effectiveness and the their biological activities. \\
& In addition, (QSAR) data has been used to develop relationships between \\
& biological activities and thermophysical properties of chemicals, through the \\
& Hyper Chem 8.0 program by using Semi-empirical (SE) method at the (PM3) \\
& level. The LOG P value was calculated, binding energy, Polarizability, hydration \\
& energy, surface area, and electrostatic potential energy difference of two level.
\end{tabular}

DOI: $10.22401 /$ ANJS.24.2.01

*Corresponding author: Amartuma1975@gmail.com

\section{Introduction}

The benzimidazoles are potent, orally active and safe, it is a colorless solid, molecular Formula $\mathrm{C}_{7} \mathrm{H}_{6} \mathrm{~N}_{2}$, broadspectrum anthelmintics widely used in human and veterinary Medicine. This compound is bicyclec in nature which comprises of the combination of Benzene and imidazole. The most important nitrogen-containing rings are Benzimidazole rings. Heterocyclic compounds hold a central role in medicinal chemistry and are important in the pharmaceutical industry's quest for new bioactive molecules because they contain heterocycles.

We hope that this paper will provide fresh opportunities for researchers to develop new and more potent benzimidazole-containing drugs for future generations. [1]. An imidazole ring can be used in many medicines, and it has become a popular component in synthetic pharmaceuticals, being an ionizing and aromatic compound, it activates pharmacokinetic properties, it are present in many antiprotozal, antifungal, and antihypertensive of antibiotics, and other [2,3]. These molecules are effective against various strains of microorganisms due to their enormous medicinal value,
Imidazole is contained in the anticancer drug mercaptopurine, which acts by interfering with DNA activities to combat leukemia [4], thus showing a wide range of vital activities and the explored and used on a large scale by the pharmaceutical industry.

\section{Biological Activity}

Pharmacological biological activity plays a crucial role as it recommends the use of compounds in medical applications, because biological reactions of living organisms, biological activity, or pharmacological activity describe the beneficial or harmful effects of a drug on a living substance, chemical compounds may exhibit some harmful and toxic effects that may prevent their use in practice medical [5], this activity is exercised by the active ingredient of the substance or drug carrier, but it can be modified by the other ingredients [6]. Activity is generally dose dependent. Moreover, the effects ranging from beneficial to harmful for a single substance are when the amount of doses is changed from low to high. The compound must have the appropriate properties (absorption, distribution, metabolism, and excretion) 


\section{Al-Nahrain Journal of Science}

ANJS, Vol.24 (2), June, 2021, pp. 1-8

necessary to add its bioactivity to make it suitable for use as a drug [7].

\section{Quantum Chemical}

Usually the compound is filtered for use as medicine. Starting with formulations, all of their thermochemical and biochemical properties are synthesized for use in experimental testing. These are time consuming and cost effective and therefore computational chemistry has been used, by which a lot of drugs are designed to discover chemical, biochemical, and physiochemical properties. It makes use of DFT is a very useful tool for predicting experimental outcomes, evaluating chemical properties of molecules, and significant reforms in molecules, particularly when combined with the B3LYP mode, which produces results that are very similar to observational data.

In addition, quantitative structure activity relationships (QSAR) drug research and growth also relied on establishing interactions between biological processes and physicochemical properties of chemical compounds. Work is being done on constructing the spatial chemical composition of each measured molecule, beginning by drawing the molecule's $2 \mathrm{D}$ structure bit by bit. The hydrogen atoms are then immediately inserted, Second, the chemical construction and structure option is converted into a three-dimensional structure.

The theoretical properties of the compound being studied are recorded through some parameters such as Free Energy, Entropy, Dipole Moment, Binding Energy, nuclear power, Electronics Energy, formation heat, HOMO, LUMO. By using the DFT method was fixed via 6-311 G, and B3-LYP [9], by Gaussian 09 program. The Charge Density, Grid Surface Area, Volume, Log P, Polarization, Refractivity, and Molecular Mass are all measured as QSAR properties of particles, through Hyper Chem 8.0 program by using Semi-Empirical (SE) method at the (PM3) level.

\section{Results and Discussion}

\subsection{Optimized structure}

The optimized structure of optimized molecules of some benzimidazole derivatives \{Carbenzim (CZM), Mebendazole (MBZ)\} using Gaussian 09 software, their plane of Symmetry and inconsistency were found by improving the structural molecules mentioned in Table 1, illustrated in Figure 1.
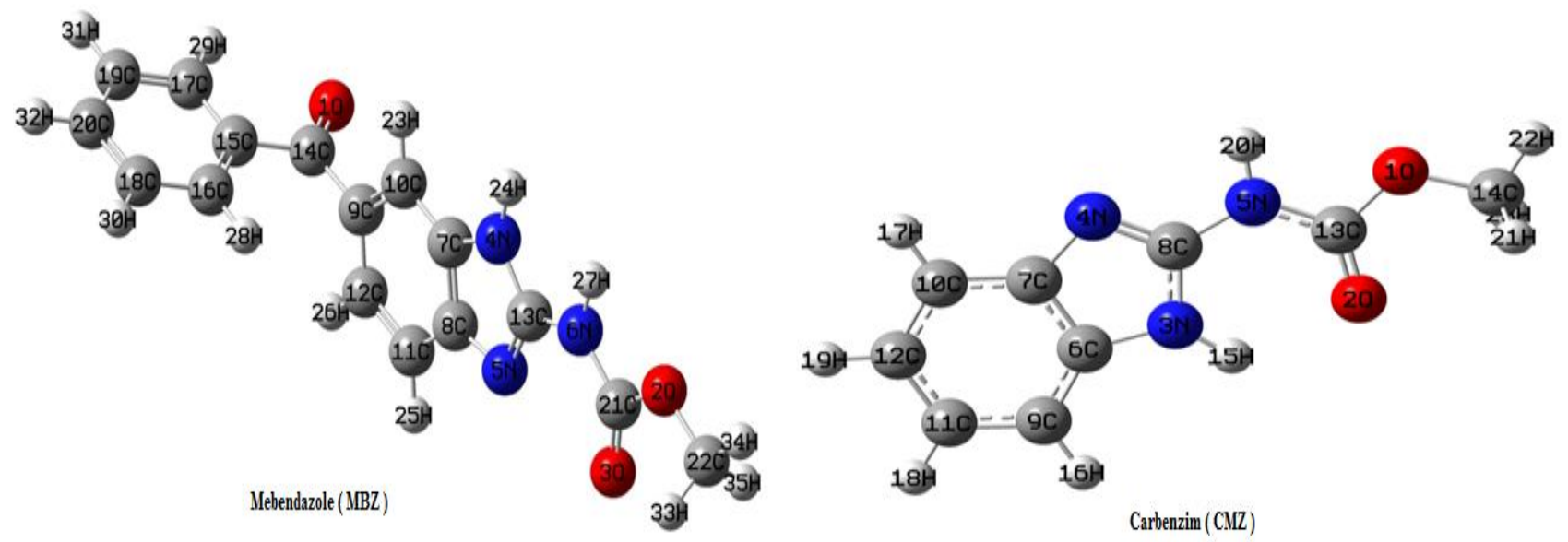

Figure 1. Optimized molecular structure for $\{$ Carbenzim (CZM), Mebendazole (MBZ) $\}$. 


\section{Al-Nahrain Journal of Science}

ANJS, Vol.24 (2), June, 2021, pp. 1-8

Table 1. The optimized geometry data of $\{(\mathrm{CZM}),(\mathrm{MBZ})\}$ molecule for Bond length and Bond angle.

\begin{tabular}{|c|c|c|c|c|c|c|c|}
\hline $\begin{array}{l}\text { Structural } \\
\text { parameter }\end{array}$ & $\begin{array}{c}\text { DFT/ } \\
\text { 6-311-G } \\
\text { This work }\end{array}$ & $\begin{array}{l}\text { Structural } \\
\text { parameter }\end{array}$ & $\begin{array}{c}\text { DFT/ } \\
\text { 6-311-G } \\
\text { This work }\end{array}$ & $\begin{array}{l}\text { Structural } \\
\text { parameter }\end{array}$ & $\begin{array}{c}\text { DFT/ } \\
\text { 6-311-G } \\
\text { This work }\end{array}$ & $\begin{array}{l}\text { Structural } \\
\text { parameter }\end{array}$ & $\begin{array}{c}\text { DFT/ } \\
\text { 6-311-G } \\
\text { This work }\end{array}$ \\
\hline \multicolumn{2}{|c|}{$\begin{array}{l}\text { Bond length in } \\
\left(A^{o}\right)(C Z M)\end{array}$} & \multicolumn{2}{|c|}{$\begin{array}{l}\text { Bond angle in } \\
\text { Degree }(C Z M)\end{array}$} & \multicolumn{2}{|c|}{$\begin{array}{c}\text { Bond length in } \\
\left(A^{o}\right)(M B Z)\end{array}$} & \multicolumn{2}{|c|}{$\begin{array}{l}\text { Bond angle in } \\
\text { Degree }(M B Z)\end{array}$} \\
\hline $\mathrm{C}_{7}-\mathrm{C}_{6}$ & 1.41 & $\mathrm{C}_{14}-\mathrm{O}_{1}-\mathrm{C}_{13}$ & 116.53 & $\mathrm{C}_{14}=\mathrm{O}_{1}$ & 1.25 & $\mathrm{O}_{1}=\mathrm{C}_{14}-\mathrm{C}_{9}$ & 119.68 \\
\hline $\mathrm{C}_{10}-\mathrm{C}_{7}$ & 1.39 & $\mathrm{O}_{1}-\mathrm{C}_{13}=\mathrm{O}_{2}$ & 124.42 & $\mathrm{C}_{21}=\mathrm{O}_{3}$ & 1.24 & $\mathrm{C}_{21}-\mathrm{O}_{2}-\mathrm{C}_{22}$ & 116.61 \\
\hline $\mathrm{C}_{10}-\mathrm{C}_{17}$ & 1.08 & $\mathrm{H}_{21}-\mathrm{C}_{14}-\mathrm{H}_{22}$ & 111.11 & $\mathrm{C}_{21}-\mathrm{O}_{2}$ & 1.36 & $\mathrm{O}_{2}-\mathrm{C}_{22}-\mathrm{H}_{33}$ & 104.46 \\
\hline $\mathrm{C}_{7}-\mathrm{N}_{4}$ & 1.40 & $\mathrm{H}_{21}-\mathrm{C}_{14}-\mathrm{H}_{23}$ & 109.74 & $\mathrm{C}_{22}-\mathrm{O}_{2}$ & 1.47 & $\mathrm{O}_{2}-\mathrm{C}_{22}-\mathrm{H}_{35}$ & 110.04 \\
\hline $\mathrm{C}_{6}-\mathrm{N}_{3}$ & 1.39 & $\mathrm{H}_{22}-\mathrm{C}_{14}-\mathrm{O}_{1}$ & 104.50 & $\mathrm{C}_{22}-\mathrm{H}_{33}$ & 1.08 & $\mathrm{O}_{3}=\mathrm{C}_{21}-\mathrm{O}_{2}$ & 124.66 \\
\hline $\mathrm{C}_{8}=\mathrm{N}_{4}$ & 1.32 & $\mathrm{H}_{23}-\mathrm{C}_{14}-\mathrm{O}_{1}$ & 110.12 & $\mathrm{C}_{21}-\mathrm{N}_{6}$ & 1.37 & $\mathrm{O}_{3}=\mathrm{C}_{21}-\mathrm{N}_{6}$ & 125.18 \\
\hline $\mathrm{C}_{8}-\mathrm{N}_{3}$ & 1.37 & $\mathrm{O}_{1}-\mathrm{C}_{13}-\mathrm{N}_{5}$ & 110.19 & $\mathrm{~N}_{6}-\mathrm{H}_{27}$ & 1.00 & $\mathrm{H}_{33}-\mathrm{C}_{22}-\mathrm{H}_{34}$ & 111.16 \\
\hline $\mathrm{N}_{3}-\mathrm{H}_{15}$ & 1.00 & $\mathrm{O}_{2}=\mathrm{C}_{13}-\mathrm{N}_{5}$ & 125.38 & $\mathrm{C}_{13}-\mathrm{N}_{6}$ & 1.38 & $\mathrm{C}_{21}-\mathrm{N}_{6}-\mathrm{H}_{27}$ & 119.01 \\
\hline $\mathrm{C}_{8}-\mathrm{N}_{5}$ & 1.39 & $\mathrm{C}_{13}-\mathrm{N}_{5}-\mathrm{H}_{20}$ & 119.02 & $\mathrm{C}_{13}-\mathrm{N}_{4}$ & 1.37 & $\mathrm{H}_{27}-\mathrm{N}_{6}-\mathrm{C}_{13}$ & 115.98 \\
\hline $\mathrm{N}_{5}-\mathrm{H}_{20}$ & 1.00 & $\mathrm{H}_{20}-\mathrm{N}_{5}-\mathrm{C}_{8}$ & 115.83 & $\mathrm{C}_{13}=\mathrm{N}_{5}$ & 1.32 & $\mathrm{C}_{21}-\mathrm{N}_{6}-\mathrm{C}_{13}$ & 124.99 \\
\hline $\mathrm{C}_{13}-\mathrm{N}_{5}$ & 1.36 & $\mathrm{C}_{8}-\mathrm{N}_{4}-\mathrm{C}_{7}$ & 104.34 & $\mathrm{~N}_{4}-\mathrm{H}_{24}$ & 1.00 & $\mathrm{~N}_{6}-\mathrm{C}_{13}=\mathrm{N}_{5}$ & 122.84 \\
\hline $\mathrm{C}_{13}-\mathrm{O}_{1}$ & 1.37 & $\mathrm{~N}_{4}-\mathrm{C}-\mathrm{C}_{6}$ & 110.04 & $\mathrm{C}_{7}-\mathrm{N}_{4}$ & 1.39 & $\mathrm{~N}_{6}-\mathrm{C}_{13}-\mathrm{N}_{4}$ & 123.02 \\
\hline $\mathrm{C}_{13}=\mathrm{O}_{2}$ & 1.24 & $\mathrm{C}_{7}-\mathrm{C}_{6}-\mathrm{N}_{3}$ & 105.11 & $\mathrm{C}_{8}-\mathrm{N}_{5}$ & 1.40 & $\mathrm{C}_{13}-\mathrm{N}_{4}-\mathrm{H}_{24}$ & 123.73 \\
\hline \multirow[t]{10}{*}{$\mathrm{C}_{14}-\mathrm{O}_{1}$} & 1.46 & $\mathrm{C}_{6}-\mathrm{N}_{3}-\mathrm{C}_{8}$ & 106.34 & $\mathrm{C}_{7}-\mathrm{C}_{8}$ & 1.42 & $\mathrm{H}_{24}-\mathrm{N}_{4}-\mathrm{C}_{7}$ & 129.91 \\
\hline & & $\mathrm{N}_{3}-\mathrm{C}_{8}-\mathrm{N}_{4}$ & 114.14 & $\mathrm{C}_{8}-\mathrm{C}_{11}$ & 1.39 & $\mathrm{C}_{13}-\mathrm{N}_{4}-\mathrm{C}_{7}$ & 106.35 \\
\hline & & $\mathrm{C}_{8}-\mathrm{N}_{3}-\mathrm{H}_{15}$ & 123.63 & & & $\mathrm{~N}_{4}-\mathrm{C}_{7}-\mathrm{C}_{8}$ & 105.00 \\
\hline & & $\mathrm{H}_{15}-\mathrm{N}_{3}-\mathrm{C}_{6}$ & 130.01 & & & $\mathrm{C}_{7}-\mathrm{C}_{8}-\mathrm{N}_{5}$ & 110.17 \\
\hline & & $\mathrm{N}_{4}-\mathrm{C}_{7}-\mathrm{C}_{10}$ & 129.79 & & & $\mathrm{C}_{8}-\mathrm{N}_{5}=\mathrm{C}_{13}$ & 104.33 \\
\hline & & $\mathrm{N}_{3}-\mathrm{C}_{6}-\mathrm{C}_{9}$ & 132.71 & & & $\mathrm{~N}_{5}=\mathrm{C}_{13}-\mathrm{N}_{4}$ & 114.12 \\
\hline & & $\mathrm{C}_{6}-\mathrm{C}_{7}-\mathrm{C}_{10}$ & 120.154 & & & $\mathrm{~N}_{5}-\mathrm{C}_{8}-\mathrm{C}_{11}$ & 129.94 \\
\hline & & $\mathrm{C}_{7}-\mathrm{C}_{10}-\mathrm{C}_{12}$ & 117.99 & & & $\mathrm{~N}_{4}-\mathrm{C}_{7}-\mathrm{C}_{10}$ & 132.62 \\
\hline & & $\mathrm{C}_{6}-\mathrm{C}_{9}-\mathrm{H}_{16}$ & 121.91 & & & $\mathrm{C}_{7}-\mathrm{C}_{8}-\mathrm{C}_{11}$ & 119.88 \\
\hline & & $\mathrm{H}_{18}-\mathrm{C}_{11}-\mathrm{C}_{12}$ & 119.32 & & & $\mathrm{C}_{11}-\mathrm{C}_{12}-\mathrm{C}_{9}$ & 121.71 \\
\hline
\end{tabular}

\subsection{Vibrational analysis} Carbendazim (czm)

The calculated vibrational using the DFT process, frequencies were calculated using the Gaussian 5 program are listed in Table 2 for Carbendazim molecule. The Carbendazim molecule consists 23 atoms, therefore it has 63 vibrational normal modes, these modes are spread representation under Cs symmetry as:

$$
\begin{aligned}
\Gamma_{\text {vib }} & =(3 \times 23 \text { atoms })-6 \\
& =22 \mathrm{~A}^{\prime \prime}+41 \mathrm{~A}^{\prime}=63 .
\end{aligned}
$$

\section{Mebendazole (MBZ)}

The Mebendazole molecule consists 35 atoms, therefore it has 99 vibrational normal modes ( 99 A) these modes are spread representation under $\mathrm{C} 1$ symmetry shown below. The calculated vibrational frequencies by Gaussian 5 program using DFT method are listed in Table 3 for Mebendazole molecule.

$$
\begin{gathered}
\Gamma_{\text {vibration }}=\Gamma_{\text {total }}-\left(\Gamma_{\text {rotation }}+\Gamma_{\text {translation }}\right) \\
=3 \mathrm{~N}-6
\end{gathered}
$$

$=(3 \times 35$ atoms $)-6=99 \mathrm{~A}$

The IR spectrum of molecules $\{$ Carbendazim (CZM), Mebendazole (MBZ) $\}$ are depicted as shown in Figure 2. 


\section{Al-Nahrain Journal of Science}

ANJS, Vol.24 (2), June, 2021, pp. 1-8

Table 2. The theoretical vibrational frequencies $\left(\mathrm{cm}^{-1}\right)$, with its assignment for (CZM) molecule.

\begin{tabular}{|c|c|c|c|c|}
\hline \multirow{2}{*}{ No. } & \multirow{2}{*}{ Sym } & \multicolumn{2}{|c|}{ This Work } & \multirow{2}{*}{ Assignment } \\
\hline & & Freq. $\left(\mathrm{cm}^{-1}\right)$ & IR Intensity $\mathrm{Km} \mathrm{mol}^{-1}$ & \\
\hline$v_{1}$ & $\mathrm{~A}^{\prime}$ & 3558.68 & 77.98 & N-H Str. \\
\hline$v_{2}$ & $\mathrm{~A}^{\prime}$ & 3548.65 & 127.2 & N-H Str. (imidazol) \\
\hline$v_{3}$ & $\mathrm{~A}^{\prime}$ & 3141.12 & 21.42 & C-H Sym. Str. (Benzimidazol) \\
\hline$v_{4}$ & $\mathrm{~A}^{\prime}$ & 3129.52 & 33.48 & C-H Asym. Str. (Benzimidazol) \\
\hline$v_{5}$ & $\mathrm{~A}^{\prime}$ & 3116.68 & 13.61 & C-H Asym. Str. \\
\hline$v_{6}$ & $\mathrm{~A}^{\prime}$ & 2993.37 & 33.29 & C-H Sym. Str. \\
\hline$v_{7}$ & $A^{\prime}$ & 1654.08 & 283.59 & $\begin{array}{c}\mathrm{C}=\mathrm{O} \text { Str, } \mathrm{C}-\mathrm{C}-\mathrm{C} \text { Str (Benzimidazol), } \\
\delta \text { Benzimidazol }+\delta \mathrm{N}-\mathrm{H} \text { (imidazol) }\end{array}$ \\
\hline$v_{8}$ & $\mathrm{~A}^{\prime}$ & 1500.59 & 47.59 & $\delta$ C-H Scis. \\
\hline$v_{9}$ & $\mathrm{~A}^{\prime}$ & 1459.85 & 2.89 & $\delta \mathrm{C}-\mathrm{H}$ Rock., $\delta \mathrm{N}-\mathrm{H}$ \\
\hline$v_{10}$ & $A^{\prime}$ & 1410.41 & 191.64 & $\begin{array}{c}\mathrm{N}-\mathrm{C}-\mathrm{N} \text { Str (imidazol), } \\
\delta \mathrm{C}-\mathrm{H} \text { Benzimidazol, C=N Str. }\end{array}$ \\
\hline$v_{11}$ & $\mathrm{~A}^{\prime}$ & 1220.15 & 121.70 & $\delta$ Benzimidzol, $\delta \mathrm{N}-\mathrm{H}, \delta \mathrm{C}-0$ \\
\hline$v_{12}$ & $\mathrm{~A}^{\prime}$ & 1178.78 & 107.41 & $\delta \mathrm{C}-\mathrm{H}$ Scis, $\delta \mathrm{O}-\mathrm{C}=\mathrm{O}$ \\
\hline$v_{13}$ & $\mathrm{~A}^{\prime}$ & 987.05 & 0.01 & $\gamma \mathrm{C}-\mathrm{H}$ Benzimidazol \\
\hline$v_{14}$ & $\mathrm{~A}^{\prime \prime}$ & 1142.66 & 0.40 & $\gamma \mathrm{C}-\mathrm{H}$ Twis. \\
\hline$v_{15}$ & $\mathrm{~A}^{\prime \prime}$ & 732.80 & 123.0 & $\begin{array}{c}\gamma \mathrm{N}-\mathrm{H}(\text { Imidazol), } \gamma \mathrm{N}-\mathrm{H}, \\
\gamma \mathrm{C}-\mathrm{H} \text { Benzimidazol, } \gamma \mathrm{C}=\mathrm{O}\end{array}$ \\
\hline$v_{16}$ & $\mathrm{~A}^{\prime \prime}$ & 440.51 & 2.92 & $\gamma$ Benzimidazol \\
\hline$v_{17}$ & $A^{\prime \prime}$ & 87.46 & 4.99 & $\begin{array}{c}\gamma \mathrm{C}-\mathrm{H} \text { Wag, } \gamma \mathrm{C}-\mathrm{O}, \\
\gamma \text { Benzimidazol, } \gamma \mathrm{N}-\mathrm{H}, \gamma \mathrm{C}=\mathrm{O}\end{array}$ \\
\hline
\end{tabular}

Table 3. The theoretical vibrational frequencies $\left(\mathrm{cm}^{-1}\right)$, with its assignment for (MBZ) molecule.

\begin{tabular}{|c|c|c|c|c|}
\hline \multirow{2}{*}{ No. } & \multirow{2}{*}{ Sym } & \multicolumn{2}{|r|}{ This Work } & \multirow{2}{*}{ Assignment } \\
\hline & & Freq. $\left(\mathrm{cm}^{-1}\right)$ & IR Intensity $\left(\mathrm{Km} \mathrm{mol}^{-1}\right)$ & \\
\hline$v_{1}$ & A & 3557.93 & 86.78 & $\mathrm{~N}-\mathrm{H}$ Str. \\
\hline$v_{2}$ & $\mathrm{~A}$ & 3543.99 & 155.45 & N-H Str. (Imidazol) \\
\hline$v_{3}$ & $\mathrm{~A}$ & 3148.41 & 11.73 & C-H Sym. Str. (Benzimidazol), \\
\hline$v_{4}$ & $\mathrm{~A}$ & 3142.24 & 12.38 & C-H Sym. Str. (Phenyl) \\
\hline$v_{5}$ & $\mathrm{~A}$ & 3141.67 & 3.32 & C-H Asym. Str. (Benzimidazol) \\
\hline$v_{6}$ & $\mathrm{~A}$ & 3135.42 & 14.23 & C-H Asym. Str. (Phenyl) \\
\hline$v_{7}$ & $\mathrm{~A}$ & 3119.40 & 13.48 & C-H Asym. Str. \\
\hline$v_{8}$ & $\mathrm{~A}$ & 2995.57 & 33.48 & $\begin{array}{l}\text { C-H Sym. Str. } \\
\end{array}$ \\
\hline$v_{9}$ & $\mathrm{~A}$ & 1657.07 & 216.6 & $\mathrm{C}=\mathrm{O}$ Str. (Amide), $\mathrm{C}-\mathrm{C}-\mathrm{C}$ Str.(Benzimidazol) \\
\hline$v_{10}$ & $\mathrm{~A}$ & 1608.87 & 3.16 & C-C-C Str. (Phenyl) \\
\hline$v_{11}$ & $\mathrm{~A}$ & 1592.52 & 436.08 & $\delta \mathrm{N}-\mathrm{H}$ (Imidazol), $\delta \mathrm{N}-\mathrm{H}$ \\
\hline$v_{12}$ & $\mathrm{~A}$ & 1545.96 & 335.43 & $\mathrm{C}=\mathrm{O}$ Str. (Keton) \\
\hline$v_{13}$ & $\mathrm{~A}$ & 1508.76 & 42.25 & $\delta \mathrm{C}-\mathrm{H}$ (Phenyl), $\delta \mathrm{N}-\mathrm{H}$ (Imidazol),$\delta \mathrm{N}-\mathrm{H}$ \\
\hline$v_{14}$ & $\mathrm{~A}$ & 1499.83 & 50.70 & $\delta \mathrm{CH}_{2}$ (Scis.) \\
\hline$v_{15}$ & $\mathrm{~A}$ & 1479.78 & 57.70 & $\delta \mathrm{C}-\mathrm{H}$ (Benzimidazol) \\
\hline$v_{16}$ & $\mathrm{~A}$ & 1456.37 & 20.20 & $\delta \mathrm{CH}_{2}$ (Rock.) \\
\hline$v_{17}$ & $\mathrm{~A}$ & 1143.0 & 0.43 & $\gamma \mathrm{CH}_{2}$ (Twis.) \\
\hline$v_{18}$ & $\mathrm{~A}$ & 1017.06 & 4.95 & $\gamma$ C-H (Phenyl) \\
\hline$v_{19}$ & $\mathrm{~A}$ & 995.99 & 34.01 & $\delta$ (Imidazol) (breathing), $\delta$ (Phenyl) (Breathing) \\
\hline$v_{20}$ & $\mathrm{~A}$ & 981.01 & 1.75 & $\gamma \mathrm{C}-\mathrm{H}($ Benzimidazol) \\
\hline$v_{21}$ & $\mathrm{~A}$ & 921.74 & 7.63 & $\delta \mathrm{C}=\mathrm{O}$ \\
\hline$v_{22}$ & A & 763.50 & 39.61 & $\gamma \mathrm{N}-\mathrm{H}$ (Imidazol) \\
\hline$v_{23}$ & A & 740.11 & 192.32 & $\gamma \mathrm{N}-\mathrm{H}$ \\
\hline$v_{24}$ & $\mathrm{~A}$ & 696.77 & 4.32 & $\delta \mathrm{C}-\mathrm{O}-\mathrm{C}, \delta \mathrm{C}-\mathrm{N}$ \\
\hline$v_{25}$ & $\mathrm{~A}$ & 688.09 & 0.07 & $\gamma \mathrm{C}=\mathrm{O}$ \\
\hline$v_{26}$ & $\mathrm{~A}$ & 654.20 & 70.04 & $\gamma \mathrm{N}-\mathrm{H}$ \\
\hline$v_{27}$ & $\mathrm{~A}$ & 627.70 & 4.51 & $\gamma \mathrm{C}-\mathrm{C}($ Benzimidazol) \\
\hline$v_{28}$ & $\mathrm{~A}$ & 239.89 & 8.11 & $\gamma \mathrm{C}-\mathrm{O}-\mathrm{C}$ \\
\hline
\end{tabular}




\section{Al-Nahrain Journal of Science}

ANJS, Vol.24 (2), June, 2021, pp. 1-8
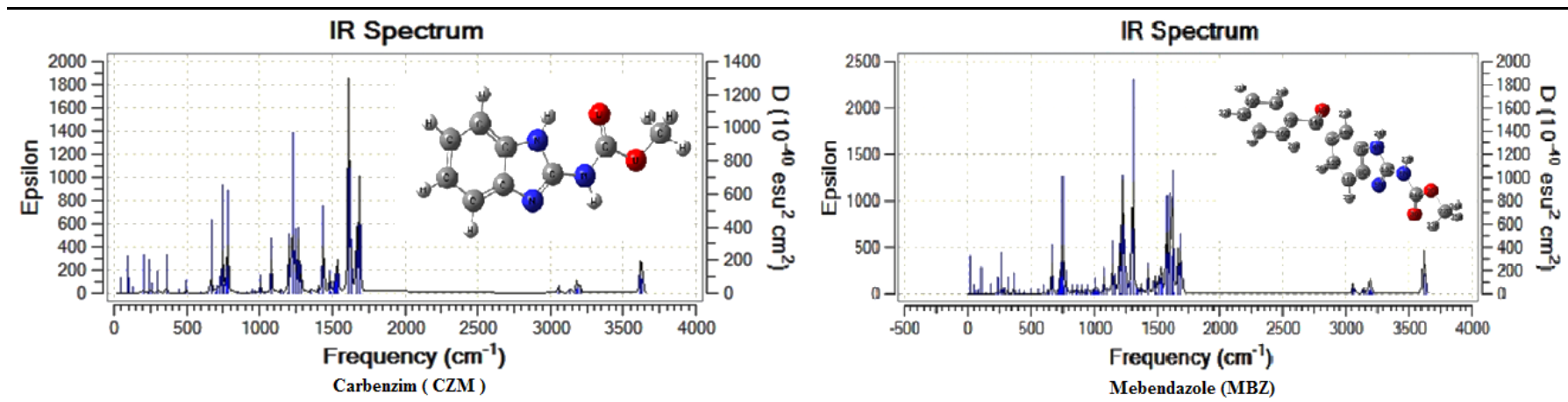

Figure 2. The calculated Infrared spectrum of $\{$ Carbendazim (CZM), Mebendazole (MBZ) $\}$ molecules.

\subsection{Molecular orbital}

For chemical properties, the energy gap is the most critical parameter. The high reactivity is described as $a$ the least value energy gap. The electrophilic (Positive charge groups or atoms green color is represented is localized on the carbon and hydrogen atoms) assault the atomic target with the largest density of orbital HOMO, whereas the nucleophilic (Atoms or entities with a negative charge red color is represented is localized on the nitrogen atoms in imidazole ring and oxygen atoms) assault LUMO that is associated with atomic high density of orbital LUMO, as shown in Figure 3.

The HOMO's energy is proportional to its ionization potential, while the LUMO's energy is proportional to its electron affinity. The energy distance, $E$ gap, is defined as the difference between HOMO and LUMO energy as per to Koopman's theorem. [10].

$$
E_{\text {gap }}=\left(E_{L U M O}-E_{\text {HOMO }}\right) \approx I P-E A
$$

From the HOMO and LUMO energy values, the ionization potential $(I)$ and electron affinity $(A)$ can be determined using equations 1 and 2. Table 4 contains information on energetic values [4].

$$
\begin{aligned}
& I=-E_{\text {Номо }} \\
& A=-E_{\text {LUMO }}
\end{aligned}
$$

Table 4. Information of energetic values of the imidazole derivatives molecules Carbendazim (CZM), Mebendazole (MBZ)\}.

\begin{tabular}{||c|c|c|}
\hline Parameter & CZM & MBZ \\
\hline \hline HOMO, $(\mathrm{ev})$ & -6.0604 & -6.3205 \\
\hline LUMO, $(\mathrm{ev})$ & -0.7950 & -1.9725 \\
\hline$\Delta \mathrm{E},(\mathrm{LUMO}-\mathrm{HOMO}$ & 5.26540 & 4.3479 \\
\hline Ionization potential (IP), ev & 6.06048 & 6.3205 \\
\hline Electron affinity (EA), ev & 0.7950 & 1.9725 \\
\hline
\end{tabular}

These chemical descriptors were defined by Koopman's theory [11], by means of HOMO and LUMO energies to define universal reaction descriptors in order to define molecular or atomic properties of interest and chemical quantities and represented by \{Electrophilicity $(\omega)$, Chemical Potential $(\mu)$, Electronegativity $(\chi)$,
Hardness $(\eta)$ and Softness $(S)\}$ in the framework of the molecular orbit expressed by relationships, according to the following equations (3), (4), (5), (6) and (7).

The data of chemical descriptors of imidazole derivatives molecules $\{$ Carbendazim (CZM), Mebendazole (MBZ) $\}$, as shown in Table 5 .

$$
\begin{aligned}
& \mu=-\frac{I+A}{2} \\
& \eta=\frac{\mathrm{I}-\mathrm{A}}{2} \\
& S=\frac{I}{\eta} \\
& \chi=\frac{I+A}{2} \\
& \omega=\frac{\mu}{2 \eta}
\end{aligned}
$$

Table 5. Data of energetic values and data for Chemical reactivity of the imidazole derivatives molecules \{Carbendazim (CZM), Mebendazole (MBZ) \}.

\begin{tabular}{||c|c|c||}
\hline Parameter & CZM & MBZ \\
\hline \hline Hardness $(\eta)$ & 2.63274 & 2.174 \\
\hline Softness $(S)$ & 0.379832 & 0.45998 \\
\hline Electrophilicity $(\omega)$ & 2.23140 & 3.95433 \\
\hline Chemicalpotential $(\mu)$ & -3.42774 & -4.1465 \\
\hline Electronegativity $(\chi)$ & 3.42774 & 4.1465 \\
\hline
\end{tabular}




\section{Al-Nahrain Journal of Science}

ANJS, Vol.24 (2), June, 2021, pp. 1-8
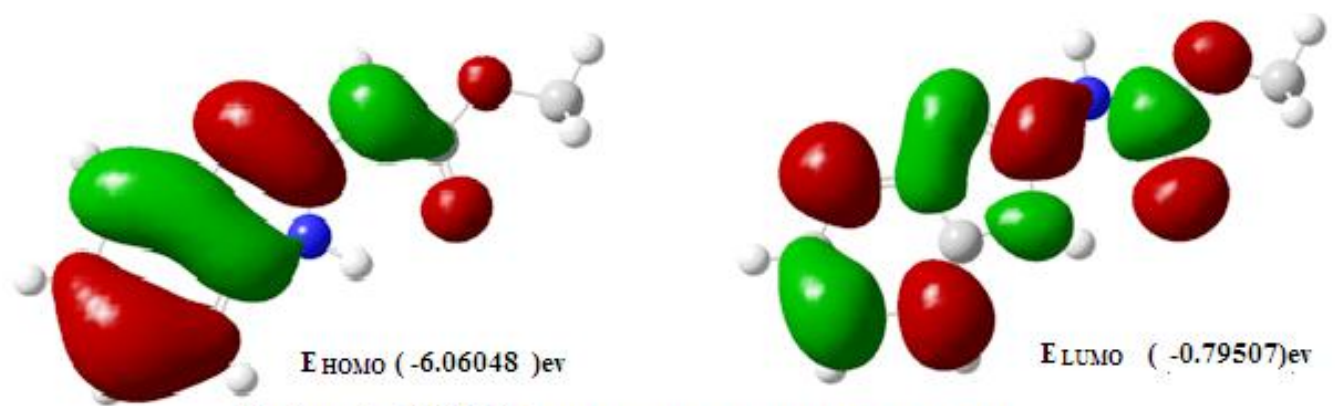

Carbenzim $(\mathrm{CZM}): \mathrm{E}_{\text {gap }}=\mathrm{E}_{\text {LUMO }}-\mathrm{E}_{\text {Hoмо }}=(\mathbf{5 . 2 6 5 4 0}) \mathrm{er}$

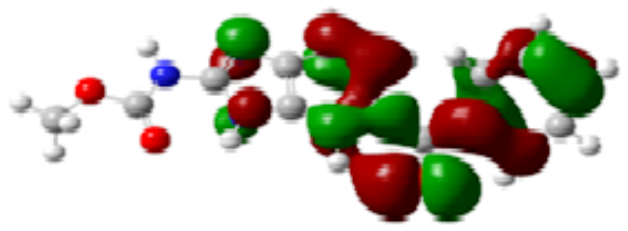

E hомо $(-6.3205)$ ev

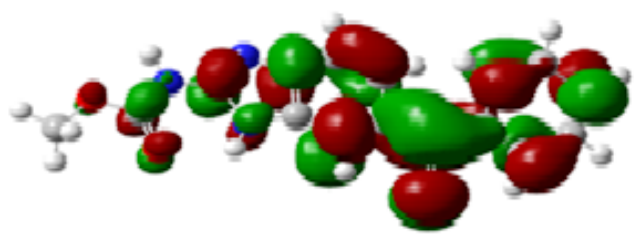

ELUMo (-1.9725 ) er

Mebendazole $(\mathrm{MBZ}): \mathrm{E}_{\text {gap }}=\mathrm{E}_{\text {LUMO }}-\mathrm{E}_{\text {Hомо }}=(4.34793) \mathrm{ev}$

Figure 3. The energy values with molecular orbital geometry of $\{(\mathrm{CZM}),(\mathrm{MBZ})\}$ molecules in gas phase.

\subsection{Thermophysical properties}

The binding energy of a drug is an important parameter to consider when developing a new drug. It is better to have a large value negative for drugs, where the value is more negative. This gives an indication that the molecule is a good drug. The molecule with the lowest (Binding Energy) has the greatest (Binding Affinity).

Semi-Empirical PM3 Method of the program HyperChem 8.0 was accustomed to evaluate the thermophysical properties like dipole moment, formation energy, binding energy, and nuclear energy of imidazole derivatives $\{(\mathrm{CZM}),(\mathrm{MBZ})\}$, as shown in the Table 6 .

Table 6. Thermophysical properties.

\begin{tabular}{||c|c|c||}
\hline Properties & CZM & MBZ \\
\hline \hline Total energy $(\mathrm{kcal} / \mathrm{mol})$ & -52499.9 & -79856.9 \\
\hline Heat Capacity, $(\mathrm{kcal} / \mathrm{moldeg})$ & 0.0 & 0.0 \\
\hline Dipole Moment $(\mathrm{D})$ & 3.066 & 2.381 \\
\hline Binding Energy $(\mathrm{kcal} / \mathrm{mol})$ & -2451.66 & -3974.73 \\
\hline Heat of Formation & 13.3825 & -45.4885 \\
\hline
\end{tabular}

\section{Biological Activity of Optimized Molecules}

\subsection{Quantitative structure-activity relationships} (QSAR)

As a result, the movement of electrons in a drug molecule has a significant impact on drug behavior and distribution; the electronic distribution of drug structure can regulate drug activity and distribution. As a result, the movement of electrons in a drug molecule has a significant impact on drug behavior and distribution; the electronic distribution of drug structure can regulate drug activity and distribution.

The most important characteristics (QSAR) that affects of biological activity are :

\subsection{Hydration energy}

The lower the hydration energy, the greater the ability to dissolve in water, indicating the hydrophilic existence of the compound and predicting its best properties. The energy absorbed as a material dissolves in water is referred to as hydration energy. Note that the water energy (MBZ) $(-10.5 \mathrm{kcal} / \mathrm{mol})$ is more than $(\mathrm{CZM})(-8.8 \mathrm{kcal} / \mathrm{mol})$, as shown in Table 7 .

\subsection{Polarizability}

Molecules that are highly polarizable are likely to attract other molecules intensely. A molecule's polarizability can help it dissolve further in water, we also note that the compounds (MBZ) (31.10) more than CZM) (19.52) has the highest polarizability, as shown in Table 7.

\section{$5.4 \log P$}

$\log P$ is a metric to be used as an indicator of the drug's passage across these membranes (A drug in order to reach its site of action has to pass through a number of biological), for the compounds used. The hydrophilicity is indicated by a negative $\log P$ value, whereas the hydrophobicity is indicated by a positive $\log P$ value. Since hydrophobic medications are stored longer and have a broader range in the body, they are more dangerous. As a result, the optimal distribution coefficient for a prescription is normally somewhere in the middle (not too 


\title{
Al-Nahrain Journal of Science
}

\author{
ANJS, Vol.24 (2), June, 2021, pp. 1-8
}

hydrophobic nor too hydrophilic). For good oral bioavailability, $\log \mathrm{P}$ must be in the range $(0<\log P<3)$, [12].

Table 7. QSAR properties.

\begin{tabular}{|c|c|c||}
\hline Properties & CZM & MBZ \\
\hline \hline Gradient (kcal/mol/Ang) & 0.083 & 0.099 \\
\hline Surface area (Grid) (A02) & 385.56 & 528.43 \\
\hline Surface area (approx) (A02) & 328.05 & 437.00 \\
\hline Hydration Energy(Kcal/mol) & -8.88 & -10.50 \\
\hline Partial Charges & 0.0 & 0.0 \\
\hline Polarizability & 19.52 & 31.10 \\
\hline Log P & -0.16 & 0.92 \\
\hline
\end{tabular}

\section{The Distribution Electrostatic Potential Due to} 3D Mapped Structure

When it comes to a molecule's biological function, the surface region is a crucial factor to remember. The surface area totally determines the charged distribution from electrostatic potential. More pathogens can be destroyed by increasing the charge surface area of a molecule. The larger the biological activity, the more positive charge surface region there is.
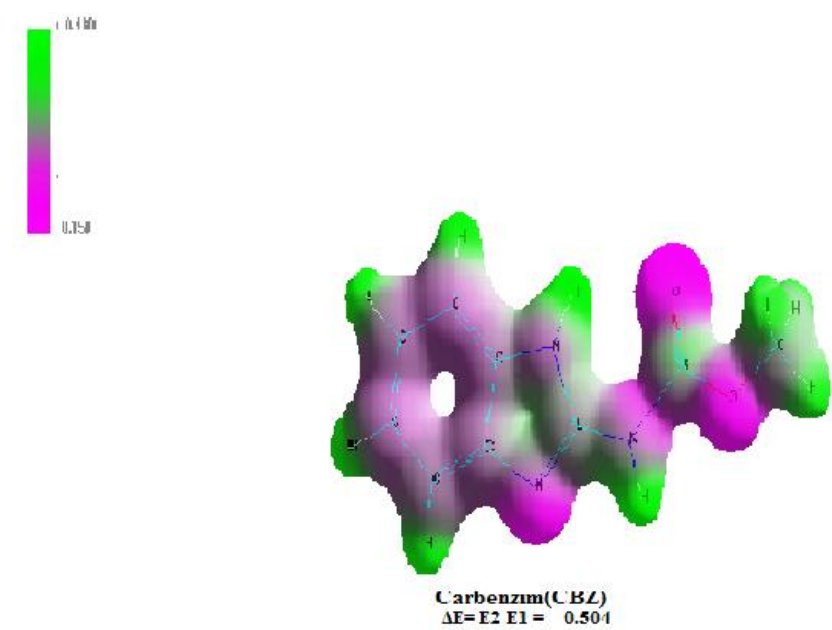

On the basis of the energy gap $\Delta \mathrm{E}$ frontier orbital, a compound's biological activity can be calculated. The positive charge end of molecules is what causes pathogen's plasma membranes to be damaged [13]. The values $(\Delta E)$ more negative the electrostatic potential in view of $3 \mathrm{D}$ mapped structure indicates regarded as the most effective methods for estimating biological activity parameters [14], extracted from the equation (8), as shown in Figure 5.

$$
\Delta E=E_{2}-E_{1}
$$

Here:

$E_{1}=$ Electrostatic Potential Energy in positive value,

$E_{2}=$ Electrostatic Potential Energy in negative,

$\Delta E=$ Electrostatic Potential Energy difference of two level.

Table 8. Information's of electrostatic potential energy of imidazole derivatives molecules \{Carbendazim (CZM), Mebendazole (MBZ) \}.

\begin{tabular}{||c|c|c||}
\hline Parameter & CZM & MBZ \\
\hline \hline$E_{1}$ & +0.378 & +0.437 \\
\hline$E_{2}$ & -0.126 & -0.172 \\
\hline$\Delta E=E_{2}-E_{1}$ & -0.504 & -0.609 \\
\hline
\end{tabular}

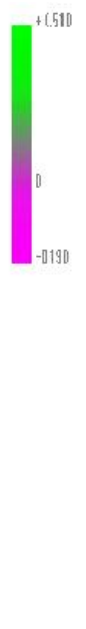

Figure 5. The 3D geometry of the distribution electrostatic potential for imidazole derivatives $\{[\mathrm{CZM}],[\mathrm{MBZ}]\}$.

\section{Conclusions}

The most notable results of this research are outlined by the following points:

1. The (MBZ) has the least gap the most critical parameter for chemical reactivity is the gap $\}$ and therefore is considered the most reactive than (CZM).

2. By comparing the values of global reactivity between compounds was observed (MBZ) more than (CZM) for each of the parameters Softness (S), Electronegativity $(\chi)$ Electrophilicity $(\omega)$, and As well (MBZ) less than (CZM) For each of the parameters Hardness $(\eta)$, Chemical potential $(\mu)$, therefore is considered (MBZ) the most reactive than (CZM).
3. The results showed that the electrostatic $(\Delta E)$ values in light of the three-dimensional structure of the two components are Converging and acceptable, and (MBZ) has the highest value (528.43) for the surface area network more than (CZM) (385.56), so it is the most active.

4. The results of values $(\log P)$ of the $(\mathrm{MBZ})$ are more acceptable than the values of the compound (CZM) and therefore is considered the good bioavailability.

\section{Thanks and Appreciation}

A special thanks to Al-Nahrain University's Department of Chemistry, College of Science, for their invaluable assistance in carrying out this study, and I am thankful to 


\section{Al-Nahrain Journal of Science}

ANJS, Vol.24 (2), June, 2021, pp. 1-8

my professor Dr. Khalida Abaid (Department of Chemistry, Al-Nahrain University) and Dr. Faryal Majeed (Veterinary Medicines Department, Industrial Research and Development Department, Ministry of Industry and Minerals)

\section{References}

[1] Anand K. and Sharad W.; "Development of drugs based on Benzimidazole Heterocycle: Recent advancement and insights", 2017.

[2] Karitzky A. R.; Rees, C. W. R. and Scriven E. F. V.; "Comprehensive heterocyclic chemistry", 5, 469-498, 1984.

[3] Grimmett M. and Ross; "Imidazole and benzimidazole synthesis", Academic Press, 1997.

[4] Hochachka P. W. and Somero G. N.; "Biochemical adaptation: mechanisms and process in physiological evolution", New York: Oxford University Press, 2002.

[5] Barker H. A.; Smyth R. D.; Weissbach H.; Toohey J. I.; Ladd J. N. and Volcani B. E.; "Isolation and properties of crystalline cobamide coenzymes containing Benzimidazole or 5,6-Dimethyl-benzimidazole", Journal of Biological Chemistry, 1960.

[6] Keane M.; "Encyclopedia \& dictionary of medicine", Nursing, and Allied Health, Seventh Edition, 2003

[7] Etymology G. K.; "Etymology, bios, life; L, activus, with energy", Mosby's Medical Dictionary, $8^{\text {th }}$ Edition, 2009.

[8] Jagan A.; Mohan R.; Manas R. B.; Gajendra L. M. and Parthasarathy T.; "Computational approach for designing and development of potent inhibitor for hepatitis-b virus $\mathrm{X}$-associated protein through molecular docking studies", 2012.

[9] Oyebamiji A. K. and Adeleke B. B.; "Theoretical investigation on biological activity of phenacetin and its derivatives via DFT and docking approach", 2018.

[10] Hossain M. I.; Kumer A.; Asian J. Phys. Chem. Sci., 3, 1-13, 2017.

[11] Koopmans T. C.; "Physica", Amsterdam, 1, 104-113 1934.

[12] ACD/Log P; "Advanced chemistry development", Inc., Toronto, Canada.

[13] Timofeeva L. and Kleshcheva N.; Appl. Microbial. Biotechnol., 89, 475-492, 2011.

[14] Böhm M.; Stürzebecher J. and Klebe G.; "Threedimensional quantitative structure-activity relationship analyses using comparative molecular field analysis and comparative molecular similarity indices analysis to elucidate selectivity differences of inhibitors binding to trypsin, thrombin, and factor Xa", Journal of Medicinal Chemistry, 42, 458-477. 1999. 